\title{
On Performance of Big Data Storage on Cloud Mechanics in Mobile Digital Healthcare
}

\author{
Abhilasha Rangra, Jaypee University of Information Technology, India \\ Vivek Kumar Sehgal, Jaypee University of Information Technology, India \\ iD https://orcid.org/0000-0002-0026-2284
}

\begin{abstract}
In recent years, the concept of cloud computing and big data analysis are considered as two major problems. It empowers the resources of computing to be maintained as the service of information technology with high effectiveness and efficiency. In the present scenario, big data is treated as one of the issues that the experts are trying to solve and finding ways to tackle the problem of handling big data analytics, how it could be managed with the technology of cloud computing and handled in the recent systems, and apart from this, the most significant issue is how to have perfect safety of big data in the cloud computing environment. In this paper, the authors mainly improve the performance of big data storage on cloud mechanics as the integration of mobile digital healthcare. The proposed framework involves the process of refining the sensitivity by using a deep learning approach. After this, it involves the step of computing or storage in the cloud-based server in an optimized manner. The experimental analysis provides a significant improvement in terms of cost, time, and accuracy.
\end{abstract}

\section{KEYWORDS}

Accuracy Analysis, Cloud Scheduling, Cost Analysis, Hybrid Optimization, Service Architecture, Time Analysis

\section{INTRODUCTION}

Like real mists or clouds which are the accretion of water particles, cloud as a term in the environment of cloud computing is the social event of frameworks (systems). For huge biological data produced by experimental technology, bioextract.org is used to share information extracts, a workflow framework. For example, the author checked the difficulties in industries with complex machine working and Big Data and introduced a framework for machine health prognostics. Advancement in mobility services and IoT brings the customers easy access to useful information from cloud computing and Big Data. Nevertheless, improvement is a must for knowledge and excellent data enabling the customer to decision making including health insurance. The customer can use the modalities of figuring limitlessly at any mentioned point. Instead of building up their very own physical establishment, the customers normally slant in the direction of a negotiator between suppliers for the web-based organization in the mechanism of cloud computing.

The customers need to pay only for the organizations they had used. The rest of the job required to be done can be moved to diminish the extraordinary weight in conveyed processing. A store of the organization is usually cared for by the frameworks which structure the cloud that acts as the reason 


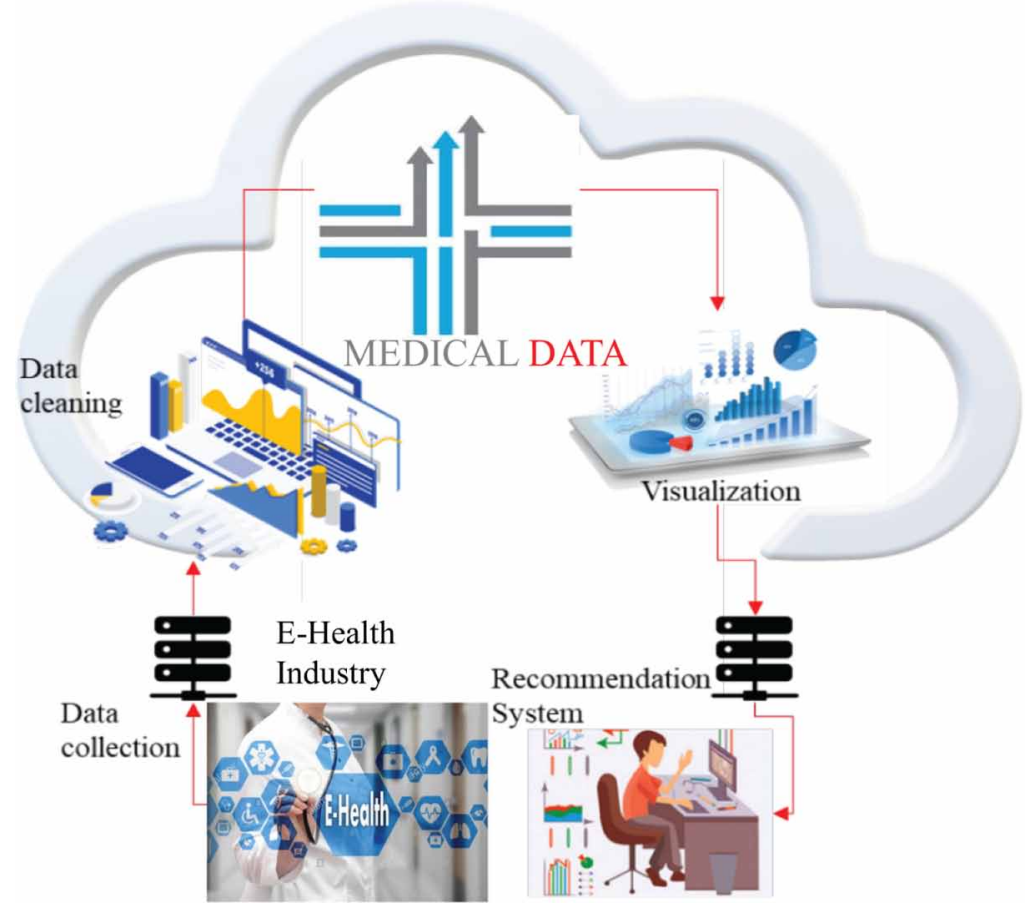

that the mass on neighborhood PCs isn't overpowering while concurrently running an application. So, at the client-side, the demand for programming and equipment is lessened. In the end, one requires a web program such as chrome to use the system of cloud-based processing and to use circulated registering. The vital high spot of computing based on clouds are:(a) Elasticity and Resource Pooling; (b) On-Demand Services and Self-Service; (c) Quality of Service (d) Pricing; (The distributed computing is administrated by three services, which are: Platform as a Service (PaaS), Software as a Service (SaaS), and Infrastructure as a Service (IaaS). The essential instances of computing that are utilized by general individuals in everyday life are YouTube, Facebook, Gmail and Dropbox, and so on. It offers adaptability, dexterity, and straightforwardness that is the reason its utilization is quickly expanding in the accomplishments (Messerli, Antony Joel, Paul Voccio, and John Carlisle Hincher, 2017, Jaisinghani, Devendra Rajkumar, 2016). There are three administrations given by the distributed form of cloud computing that is Software as a Service (SaaS), Platform as a Service (PaaS), and Infrastructure as a Service (IaaS). The essential instances of distributed computing that are utilized by general individuals in everyday life are Facebook, Dropbox, YouTube, Twitter, and Gmail, and so on. It bids versatility, adaptability, spryness, and straightforwardness that is the reason its utilization is quickly expanding in the endeavors (Shaikh, Rizwana, and M. Sasikumar, 2015).

\subsection{Cloud Computing Evolution}

In the 1960s one day in a discourse at MIT John McCarthy demonstrated that like electricity and water, registering can likewise be sold like a utility. What's more, in 1999, the Salesforce Company began dispersing the applications to the clients through an advantageous site. Amazon in 2002 begun Amazon Web Services and they were giving the administrations of capacity and calculation. In the year 2009 major organizations like Microsoft, Google, Oracle, and HP had begun to give distributed computing administrations (Stergiou, Christos, Kostas E. Psannis, Byung-Gyu Kim, and Brij Gupta, 
Figure 2. Growth of Cloud Computing Environment

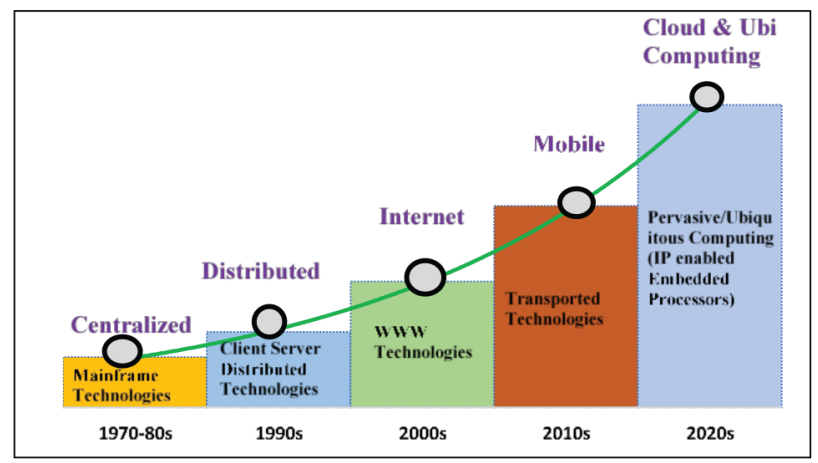

2018). These days every single individual is utilizing the administrations of cloud-based computing in their day by day life. For instance, Google Photos, Google Drive, One Drive, Dropbox, and iCloud, and so forth. In the future, distributed computing will turn into the fundamental essential of IT organizations

\subsection{Basic Components of Cloud Computing}

Three segments make up a distributed computing arrangement of cloud as delineated in Figure 3.

\subsubsection{Customers}

Clients in the design of cloud computing is expressed precisely the similar words that these are in old, plain, regular LAN (local area network). These are, ordinarily, the PCs that simply sit around your work area. Though they may likewise be workstations, tablet PCs, cell phones, or PDAs-every single enormous driver for distributed computing given their versatility. Customers act as the gadgets where the clients (end users) cooperate to deal with their data on the cloud.

\subsubsection{Datacenter}

It is the gathering of servers where the application to which you buy in is accommodated. It could be a huge room in the basement of your structure or a room brimming with servers on the opposite side of the world that you get to employ the Internet. A pattern developing in the IT world is virtualizing servers. That is, programming can be introduced enabling numerous occasions of virtual servers to be utilized. Along these lines, you can have about six virtual servers running on a solitary physical kind of server

\subsubsection{Distributed Services}

It is the situation of servers in various areas. Though the servers don't all need to be housed in a similar area. Regularly, servers are in geologically dissimilar areas. Yet, to you, the cloud supporter, these servers go about as though they're mumbling endlessly directly by one another.

\subsection{Cloud Computing Models and Services}

The three major services of cloud computing are discussed as follows.

\subsubsection{Software as a Service (SaaS)}

The method for conveying application as an administration on the web is referred to as programming as an administration commonly understood as SaaS. Instead of introducing the product on his PC, the client can get to it through the web (Shameem, P. Mohamed, and R. S. Shaji, 2013). It makes 


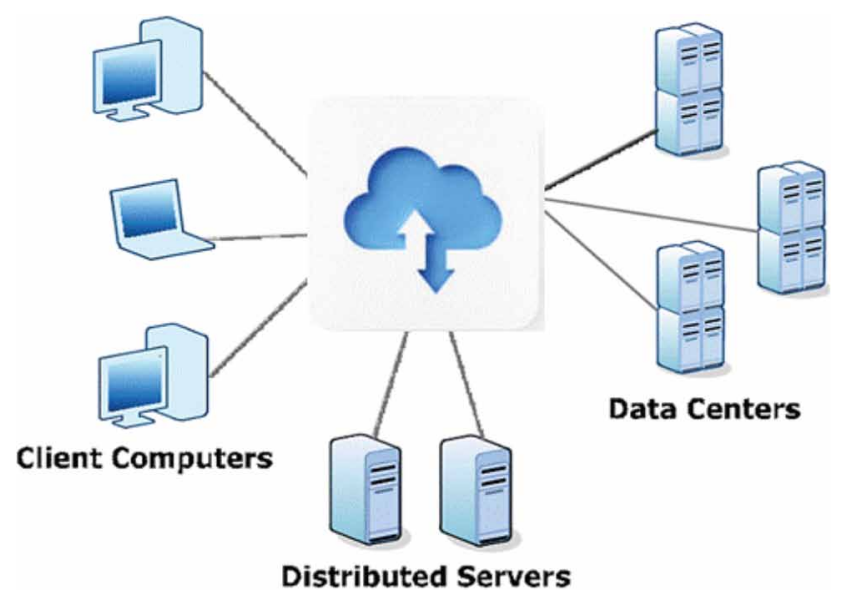

the client free from dealing with unpredictable programming and equipment. The SaaS clients don't have to purchase programming or equipment, update and maintain. The main thing client must have a web association and after that entrance to the application is extremely simple. Microsoft Office 365, Google Apps, Model, and so forth.

\subsubsection{Platform as a Service (PaaS)}

An advancement domain or stage is given to the customers as an administration in PaaS, whereupon client can send their very own product and coding. The client has the freedom to develop his very own applications that can keep running on the supplier's foundation (2019). PaaS organizations offer a predefined synthesis of the working framework and application server to acquire the administration limit of the applications. For instance, LAMP (Apache, Linux, PHP, and MySQL), Ruby, J2EE, and so forth.

\subsubsection{Infrastructure as a Service (laaS)}

Many figuring assets are given by the IaaS as storage devices, network, storage, hardware. The clients of IaaS can get to the administrations utilizing a wide zone arrange, for example, the web. For instance, a client can make virtual machines by login into the IaaS stage.

\subsubsection{Public Cloud}

The public or open cloud is a registering administration provided by the outsider suppliers on the open web (Marston, S., Li, Z., Bandyopadhyay, S., Zhang, J., Ghalsasi, A, 2011). These administrations are accessible for any client who needs to utilize them and they need to pay just for the administrations they devoured.

\subsubsection{Private Cloud}

The figuring administrations given over the web or private system go under the private cloud and these administrations are offered distinctly to the chose clients instead of average citizens (Hayes, Brian, 2008). Higher security and protection are appointed by private mists through the firewall and inward facilitating. 


\subsubsection{Hybrid Cloud}

It represents the combination of public and private clouds. In the case of a hybrid cloud structure, each one of the clouds can be coped self-reliantly but the information and applications can be shared among the clouds in the environment of a hybrid cloud. These are the mix of public as well as the private cloud. In this type of cloud, each one of the clouds can be overseen freely yet applications and information can be shared among the clouds in the hybrid environment of the cloud.

\section{RELATED WORK}

It (Srivastava, Priyanshu, and Rizwan Khan, 2018) provided an evaluation in brief for the mechanism of cloud computing by reviewing more than 30 articles on the conveyed condition of cloud computing. The aftereffect of this review signalizes the substance of the IT undertakings both after and before computing. In this, the author (Bittencourt, Luiz F., Alfredo Goldman, Edmundo RM Madeira, Nelson LS da Fonseca, and Rizos Sakellariou, 2018) introduced a scheduling problem classification in dispersed systems by bestowing a taxonomy that integrates current advances, particularly those in the environment of cloud computing. The experts have reviewed the scheduling of the literature to verify the taxonomy and examine the interest in diverse divisions of the taxonomy proposed. Lastly, identification of the relevant future directions was done in scheduling for distributed systems. It (Stergiou, Christos, Kostas E. Psannis, Byung-Gyu Kim, and Brij Gupta, 2018) presented a survey of Cc and IoT with an emphasis on the security matters of both technologies. Specifically, the experts have combined two of the abovementioned skills (i.e. IoT and Cloud Computing) for examining the common kind of features, and to determine the welfares of their incorporation. Here, the role of cloud computing in IoT technology has been presented. Thus, it displays how the technology of Cloud Computing helps in improving the IoT based function. Finally, a survey was done in conjunction with the challenges of security with the integration of Cloud Computing and IoT. In this author (Senyo, Prince Kwame, Erasmus Addae, and Richard Boateng, 2018) displayed a meta- examination of distributed research of cloud computing in data frameworks with the point of assessing writing and their related research structures, explore procedure, geological dispersion, level of investigation just as patterns of these examinations over the time of 7 years. Out of 67 audit diaries, an aggregate of 285 articles from 2009 to 2015 were utilized in the investigation. The discoveries demonstrate that surviving distributed computing writing would in general slant towards the innovative measurement to the burden of others under looked into measurements, for example, conceptualization, application area, and business. It (Hussain, Syed Asad, Mehwish Fatima, Atif Saeed, Imran Raza, and Raja

Figure 4. Classification of Cloud Computing Models

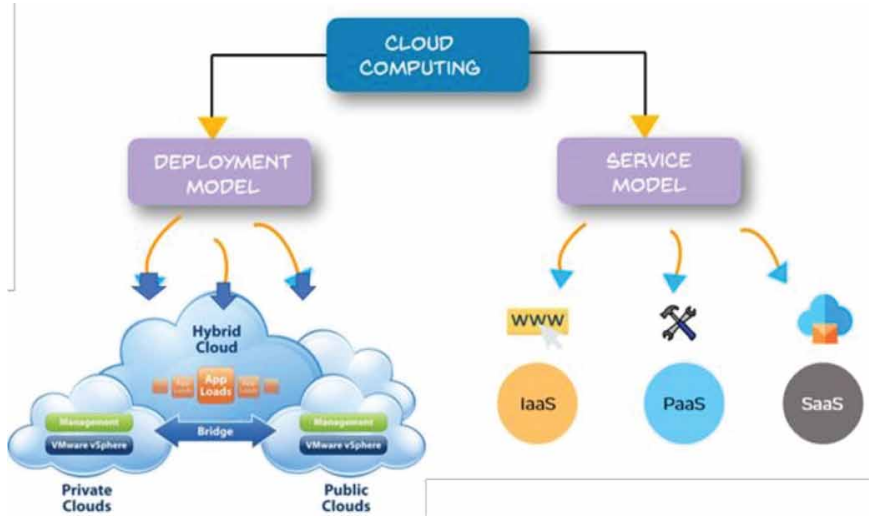


Khurram Shahzad, 2017) displayed a fresh classification (multilevel) model of numerous safety attacks crossway over numerous services of the cloud administrations at every layer. It likewise helps in distinguishing assault types and hazard levels related to various services of the cloud at the given layers. The dangers are positioned as high, low, and medium. The power of such levels of hazards relies upon the situation of clouded layers. The assaults get increasingly extreme for lower layers where framework and stage are included. The arrangement model stimulates the dynamic security arrangement contract for every layer of cloud that progressively selects prerequisites of security for cloud shoppers and suppliers. In this, it (Antony Joel Messerli et.al, 2019) introduced a first cloud controller to deal with a first cloud framework, the primary cloud foundation working a previously set of virtualized assets, the main arrangement of virtualized assets approaching the physical asset pool through the principal cloud controller. The framework further incorporates a second cloud controller to deal with a second cloud foundation, the second cloud foundation using the main arrangement of virtual assets to work the second arrangement of virtual assets, the second arrangement of virtual assets being given access to the physical asset pool during that time cloud controller and the primary cloud controller. It (Jaisinghani, Devendra Rajkumar, 2016) clarified the depiction that pursues incorporates illustrative frameworks, strategies, procedures, guidance groupings, and registering machine program items that typify the imaginative issue of the subject displayed in this work. In the accompanying portrayal, for motivations behind clarification, various explicit subtleties are gone ahead to give a comprehension of different encapsulations of the creative topic. It will be obvious, in any case, to those talented in the craftsmanship that exemplifications of the creative topic might be rehearsed. It (Hashem, Ibrahim Abaker Targio, Ibrar Yaqoob, Nor Badrul Anuar, Salimah Mokhtar, Abdullah Gani, and Samee Ullah Khan, 2015) audited ascent of enormous information in distributed computing. The definition, arrangement, and attributes of massive informational data together with certain talks on cloud-based computing were presented. The link amid massive informational data and cloud-based computing, massive informational data storing outlines, and the innovation of Hadoop were additionally talked about. Moreover, a look into difficulties was researched, with a spotlight on adaptability, accessibility, information trustworthiness, information change, information quality, information heterogeneity, security, legitimate and administrative issues, and administration. Finally, open research issues that require considerable research endeavors were condensed. It (Shaikh, Rizwana, and M. Sasikumar, 2015) proposed a grouping procedure that characterizes different parameters. The parameters were characterized dependent on different measurements. Information security can be given dependent on the level and the required insurance. Relating security arrangements at the capacity can be connected depending on the informational index usually classified as per the measurements. The productivity of the proposed grouping plan was dissected with the example of a gathered dataset. It (Shameem, P. Mohamed, and R. S. Shaji, 2013) displayed a study about powerful burden adjusting techniques coordinated on cloud information stockpiling on remaining tasks at hand. The investigation additionally centers on different measurements for burden adjusting in mists/clouds that will, in general, dissect the adequacy of existing systems. Related overhead, reaction time, execution, and versatility represent a portion of the real parameters that were considered here for investigation. In this it (Garrison, G., Kim, S., Wakefield, R.L, 2012) talked about the cloud-seller connections described by the trust that was basic for cloud arrangement and the guarantee of picking up a bit of flexibility in an aggressive market. In fruitful cloud sending, associations had the option to concentrate on the center capabilities that outcome in a competitive benefit. Associations accomplish more noteworthy IT scale economies with distributed computing when putting first in social, specialized, and administrative abilities. It (Marston, S., Li, Z., Bandyopadhyay, S., Zhang, J., Ghalsasi, A, 2011) recognized the inadequacies, potentials, starts, and risks for the industry of cloud computing. The experts have recognized diverse problems that affect various followers of the distributed mechanism of cloud computing. The specialists have likewise examined the problems, for example, a lot of proposals for the professionals who will deal with such kind of invention. At long last, scientists have laid out a share of the significant issues 
opposing administrative organizations who, due to their special idea of the invention, must turn out to be engaged personally with the guideline of distributed computing. It (Hayes, Brian, 2008) talked about the matter of distributed computing bringing up the issues about protection, security, and unwavering quality a noteworthy topic of dialog at a workshop held at Princeton University. Permitting outsider support of taking guardianship of individual reports brings up cumbersome issues about control and responsibility for cloud condition. The rest of the paper is organized as follows: the proposed method and results are discussed in Section 3 and 4 respectively, followed by a conclusion in Section 5.

\section{THE PROPOSED METHOD}

Figure 5 shows the proposed architecture. In which two-way communication between client and server. A Server makes a cloud system. Data collection, classification of sensitive and non-sensitive data done on Client-Side, and cloud side. The classification part pre-processes the text and extracts features by TF-IDF then learning by different classifiers and classifier model classified in sensitive and non-sensitive data.

The main objective of the classifier part is reducing the error and improve accuracy. The last module which performs on cloud optimize the scheduling of task and reducing cost and time at the cloud side. Fig. 6 presents the flow of the proposed methodology, which mainly improves the process of cloud scheduling using hybrid optimization. In this workflow, firstly, the text is collected, and then it is pre-processed and it extracts the labels of featuring of text using senti score function.

The function of sent score text is divided into three types of classes i.e. positive, negative, and neutral. The positive and negative are placed in a sensitive data class and the neutral is placed in the non-sensitive class. After this step, the learning process of features and class label initiates. In this paper, we use the concept of supervised learning. After the process of classification, the collected form of sensitive data. The main objective of this framework is to initiate the process of improving the mechanism of cloud scheduling using hybrid optimization approaches:

\section{Algorithm 1. For Client-Side}

\begin{tabular}{|l|l|}
\hline Step 1: & Gather the data from the tweets from Twitter by using REST API. \\
\hline Step 2: & $\begin{array}{l}\text { Pre-process the text } \\
\text { Tokenization } \rightarrow \text { Stop word removal } \rightarrow \text { Stemming }\end{array}$ \\
\hline Step 3: & $\begin{array}{l}\text { Extraction of processed tweets in the form of n-grams where } \mathrm{n}=1 \text { or } 2 \text { or } 3 \text { respectively. The evaluation was } \\
\text { done with the help of equation } 1 \text { and } 2 \text { for bigram and } 3 \text { and } 4 \text { for trigram. }\end{array}$ \\
\hline & $P\left(S_{I} \mid S_{0} \ldots S_{I-1}\right)(1)$ \\
\hline & $P\left(S_{1}^{v}\right)=\prod_{i=1}^{v} p\left(S_{i} \mid S_{i-1}\right)(2)$ \\
\hline & $\mathrm{P}\left(S_{i} \mid S_{0} \ldots S_{i-1}\right) \approx \mathrm{P}\left(S_{i} \mid S_{i-2} S_{i-1}\right)(3)$ \\
\hline Step 4: & String all features. \\
\hline Step 5: & Reduce the error. \\
\hline
\end{tabular}


Figure 5. Proposed Architecture

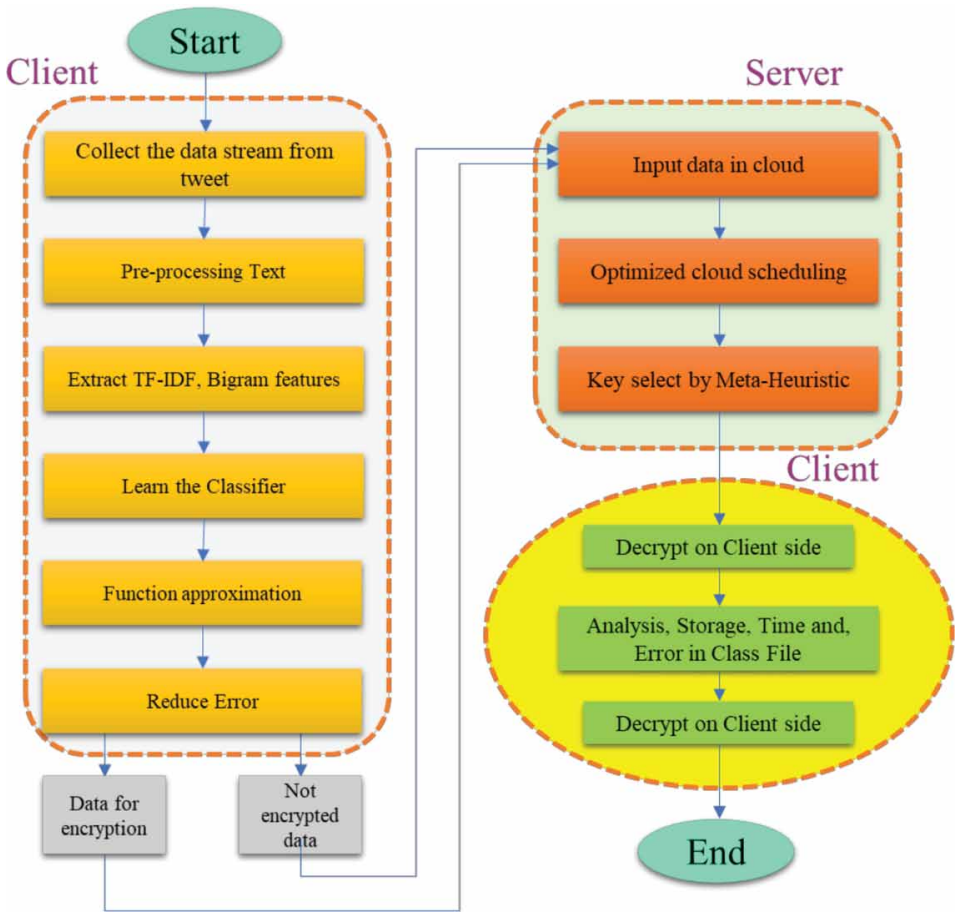

Figure 6. Cloud Scheduling Using Hybrid Optimization

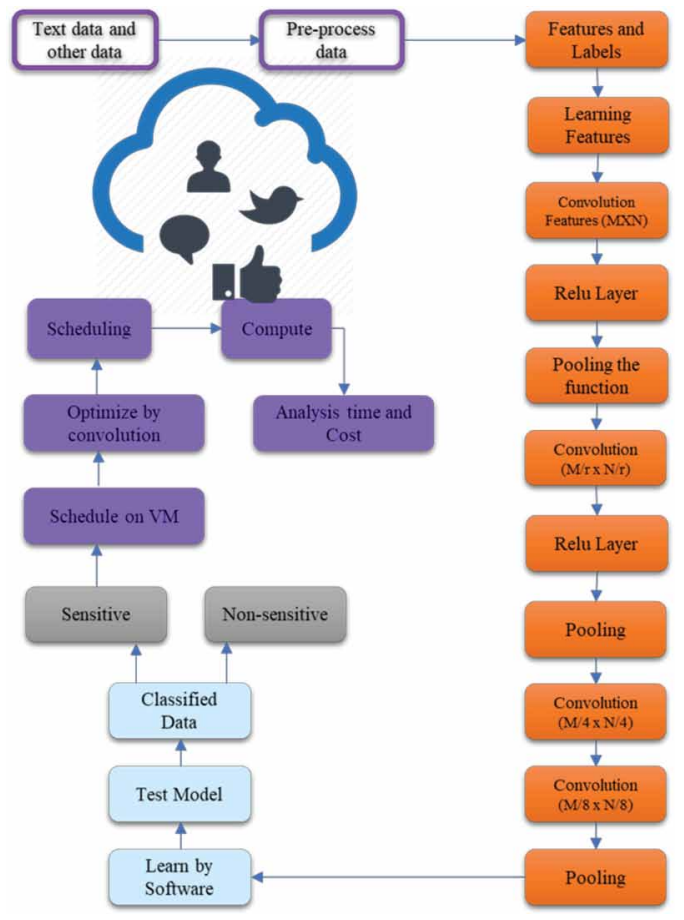


1. In the case of a single optimizer, only one work is performed at a time. But in the case of a hybrid approach, two processes work on a simultaneous basis. In our problem, we have used two processes. The first one is used to determine under-utilized virtual machines and the second involves the migration of tasks from one VM to another for the reduction of cost.

2. The process of VM migration needs local as well as global optimization. Here, only a single optimizer is not able to send the threshold in the deadline. So, it needs a single optimizer at a time.

3. But the application of a single optimizer is not converged fast that results in improper optimization known as immature exist of algorithm and random decision taken by the optimizers.

Algorithm 2. For cloud scheduling using hybrid optimization

\begin{tabular}{|l|l|}
\hline Step 1: & Internally scheduling in the cloud, the workflow and transfer the task level-wise deadline constraint \\
\hline Step 2: & Generate the matrix based on compute by the deadline, computation time, task ranking. \\
\hline Step 3: & $\begin{array}{l}\text { Hybrid (FPA-GA, FPA-PSO) } \leftarrow \text { Matrix } \\
3.1 \mathrm{~F}=\{\text { Computation, Deadline/, Budget }\} \text { presents by ranking and features vector levels } \\
3.2\left\{\left\{L_{1} \ldots L_{2}\right\}\right\} \text { Labels }\end{array}$ \\
\hline & $\begin{array}{l}3.3 \text { Input layer: } \\
A_{0}\{T, \text { Labels }\}\end{array}$ \\
\hline & $\begin{array}{l}3.4 \text { Linear layer: } \\
A_{1}\left(A_{0}\right)=W^{(1)}(f)(5)\end{array}$ \\
\hline $\begin{array}{l}3.5 \text { Activation layer: } \\
A_{2} \sigma\left(A_{1}\right)(6)\end{array}$ \\
\hline Step 4: & $\begin{array}{l}\text { Learning model check by the latest workflows and then after that we predict the ranking based on the least } \\
\text { cost and the time. }\end{array}$ \\
\hline $\begin{array}{l}\delta_{t}=\delta_{t} A_{0}^{L}(7) \\
\text { Analysis of the cost and the time of computation. }\end{array}$
\end{tabular}

\section{RESULT ANALYSIS}

In experiments analysis, the use of different numbers or quantity of data is done. The process initiates by analyzing the comparison of hybrid and single optimization approaches. The results of this analysis are based on cost parameters. Here the cost is defined using a large number of resources of task-based storage and computation. The timespan of the system presents the total processing time of all the tasks either performing the process of storage or computation. In table 1, comparative analysis of single optimizer flower pollination algorithm (FPA), Particle swarm optimization (PSO), and grey wolf optimization (GWO) is done along with analysis of hybrid optimizers i.e. FPA-GA, FPA-PSO and 
CNN. In comparison, the cost varies from 95-134 (in Rs.), which shows that the hybrid optimization effectively optimizes the cost based on earlier concepts as discussed above. In the analysis, time varies from 8543 to 13421 in milliseconds.

Time of processing improves the significance of the process but both the parameters improve in $\mathrm{CNN}$ analysis. The main reasons for the improvement in $\mathrm{CNN}$ analysis are:

1. In the CNN hybrid approach, improvement is done on by default basis.

2. In CNN, GWO semantic base optimizer finds a relation between objects and then it takes the decision. For such type of analysis, it uses alpha, Delta, and beta parameters.

3. In CNN, the methods of local and global optimization try to reduce system overhead but GWO also increases the improvement relation using semantic relation.

4. In CNN, the process of searching underutilized machines is done on a fast basis as FPA objectbased optimization uses a greedy approach.

Table 3 presents a comparison between different workflows of the proposed architecture. The first framework presents $\mathrm{CNN}$ base classification, after this process, sensitive data compute, $\mathrm{CNN}$ at cloud side scheduling (SBFG) is done. Secondly, the framework presents SVM-based classification, where the Blowfish is used for encryption same as above. But after that FPA-PSO at cloud side scheduling (SBFP) is performed, and thirdly, the same process repeats but CNN at cloud side scheduling (CNN with CONV) is performed. The fourth framework involves the use of a third framework where GWO at cloud side scheduling (SBGW) is done. The comparison of a framework using different frames as discussed above is shown in Fig. 8. This analysis indicates that CNN base framework performs well as compared to other presented frameworks as it reduces cost and time. But it provides a huge

Table 1. Algorithms based on Cost and Time

\begin{tabular}{|c|c|c|}
\hline Algorithm & Cost & Timespan \\
\hline FPA & 134 & 12343 \\
\hline PSO & 120 & 13421 \\
\hline FPA-GA & 100 & 10001 \\
\hline FPA-PSO & 102 & 9432 \\
\hline GWO & 110 & 11345 \\
\hline CNN & 95 & 8543 \\
\hline
\end{tabular}

Figure 7. (a) Cost Analysis at Cloud Side, (b) Time Analysis at Cloud Side

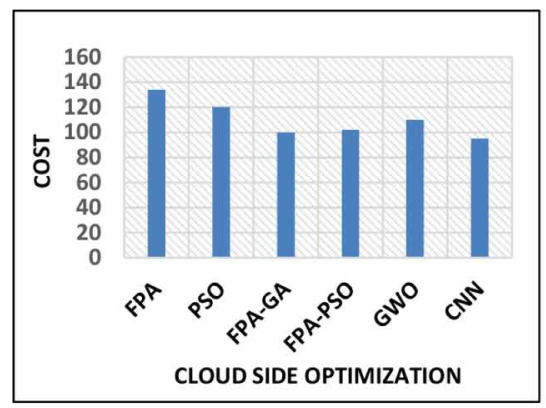

(a)

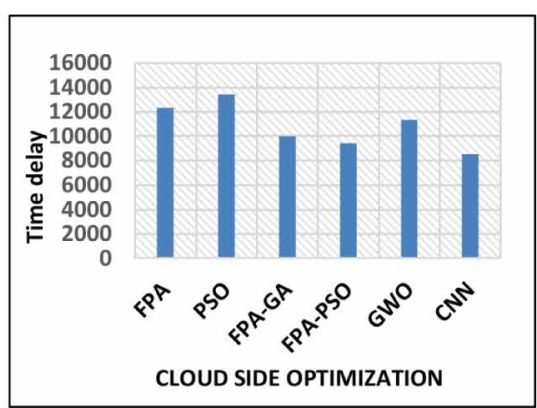

(b) 
Table 2. Comparison Framework on different parameters

\begin{tabular}{|c|c|c|c|}
\hline Framework & Cost & Timespan & Accuracy \\
\hline CNN with CONV & 96 & 12367 & 89 \\
\hline SBFP & 102 & 13245 & 90.34 \\
\hline SBFGW & 89 & 9843 & 92.34 \\
\hline SBGW & 108 & 12111 & 90.12 \\
\hline
\end{tabular}

Table 3. Size of Input based cost, time-span, accuracy, storage encryption, and time of encryption

\begin{tabular}{|l|l|l|l|}
\hline \multicolumn{1}{|c|}{ Size of Input } & \multicolumn{1}{c|}{ Cost } & \multicolumn{1}{c|}{ Timespan } & Accuracy \\
\hline 500MB & 120 & 9833 & 90.34 \\
\hline 1GB & 125 & 10324 & 94.34 \\
\hline 5GB & 203 & 12341 & 92.34 \\
\hline 10GB & 543 & 13234 & 95.45 \\
\hline 20GB & 1023 & 15323 & 96.35 \\
\hline 50GB & 1800 & 18424 & 93.23 \\
\hline 100GB & 4532 & 20335 & 95.42 \\
\hline 200GB & 10232 & 23435 & 93.45 \\
\hline
\end{tabular}

impact over accuracy as shown in Fig. 8 (c). Here, the $\mathrm{x}$-axis presents the frameworks and the $y$-axis presents accuracy.

The other parameters in the analysis of the framework involve storage encryption and time base encryption works in the same way as in CNN with CONV framework. So, we can conclude that the process of cloud scheduling affects the whole process. In this type of analysis, the effective CNN with CONV framework is selected as shown above and it performs well as compared to the other five frameworks analyzed. Table 3, Fig. 9 (a), Fig. 9 (b), and Fig. 9 (c) presents the experiment results in distinct amounts of bigdata (500MB,1GB,5GB,10GB,20GB,50GB,100GB and 200GB). The results indicate that when we increase the size of performance, the cost will increase simultaneously but it represents linear growth only that justifies this research methodology. In general, we optimize the process of big data encryption over cloud mechanics. Fig. 9 (c), show the accuracy, storage, and time performance of the proposed framework on a different number of data.

\section{CONCLUSION AND FUTURE SCOPE}

The researchers are trying their best efforts for finding an effective way to manage, maintain, process, and handle the analytics of Big data using a distinct type of parameters such as variety, velocity, and volume that requires a newly built mechanism to effectively manage, process, store, analyze and secure the big data as the process of managing and processing of big data involves various problems and it needs more effective efforts to tackle such type of requirements while dealing with analytics of big data. The mechanism of security is considered as one of the major challenges that arise when the systems try to handle and maintain the mechanism of big data. More efforts and research analysis are needed to overcome the issues of big data security instead of present methods and algorithms of security. This paper help in improving the methods of processing, storing, and analyzing the process using hybrid base optimization. The proposed framework results in better accuracy and it also helps in the reduction of time and cost. 
Figure 8. Framework-based: (a) Cost Analysis, (b) Timespan, (c) Accuracy Analysis

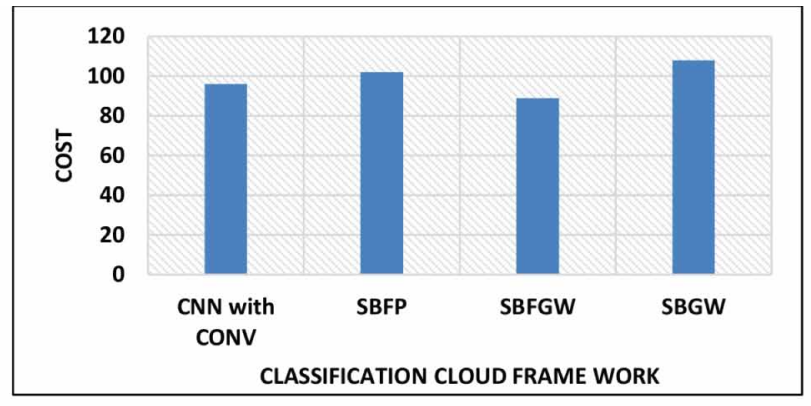

(a)

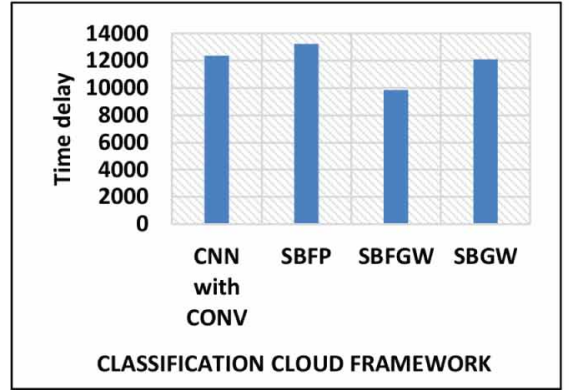

(b)

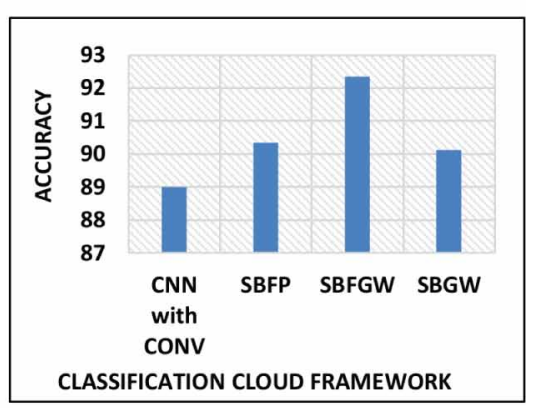

(c)

Figure 9. Size of Input-based: (a) Cost Analysis, (b) Timespan, (c) Accuracy Analysis

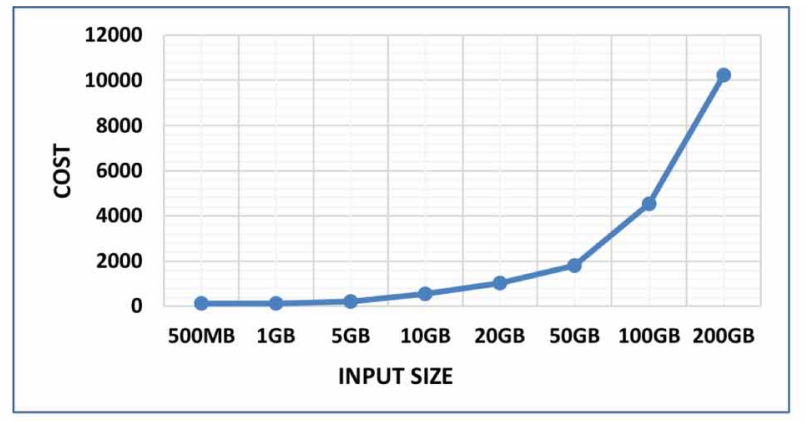

(a)

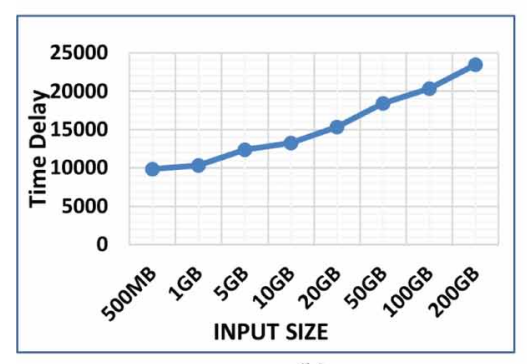

(b)

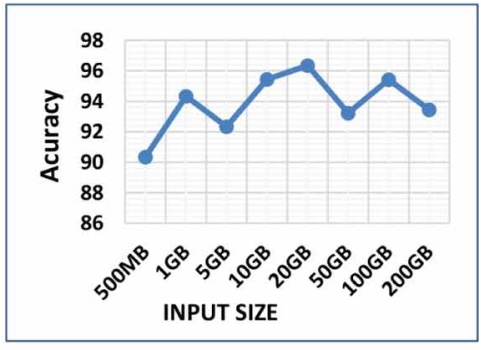

(c) 


\section{REFERENCES}

Bittencourt, L. F., Goldman, A., Madeira, E. R., da Fonseca, N. L., \& Sakellariou, R. (2018). Scheduling in distributed systems: A cloud computing perspective. Computer Science Review, 30, 31-54. doi:10.1016/j. cosrev.2018.08.002

Brian, H., Brunschwiler, T., Dill, H., Christ, H., Falsafi, B., Fischer, M., \& Kaiserswerth, M. et al. (2008). Cloud computing. Communications of the ACM, 51(7), 9-11. doi:10.1145/1364782.1364786

Cloud Computing Models. (n.d.). Available at: https://www.paranet.com/blog/bid/128265/The-Four-Types-ofCloud-Computing-Models

Cloud Computing Services. (n.d.). Available at: https://computechcorp.com/services/cloud-infrastructure-services

Components of Cloud Computing. (n.d.). Available at: https://fastcloudstorage.com/components-of-cloudcomputing

Garrison, G., Kim, S., \& Wakefield, R. L. (2012). Success factors for deploying cloud computing. Communications of the ACM, 55(9), 62-68. doi:10.1145/2330667.2330685

Hashem, I. A. T., Yaqoob, I., Anuar, N. B., Mokhtar, S., Gani, A., \& Khan, S. U. (2015). The rise of "big data" on cloud computing: Review and open research issues. Information Systems, 47, 98-115. doi:10.1016/j. is.2014.07.006

Hussain, S. A., Fatima, M., Saeed, A., Raza, I., \& Shahzad, R. K. (2017). Multilevel classification of security concerns in cloud computing. Applied Computing and Informatics, 13(1), 57-65. doi:10.1016/j.aci.2016.03.001

Jaisinghani, D. R. (2016). U.S. Patent No. 9,442,810. Washington, DC: U.S. Patent and Trademark Office.

Marston, S., Li, Z., Bandyopadhyay, S., Zhang, J., \& Ghalsasi, A. (2011). Cloud computing-The business perspective. Decision Support Systems, 51(1), 176-189. doi:10.1016/j.dss.2010.12.006

Messerli, A. J., Voccio, P., \& Hincher, J. C. (2017). U.S. Patent No. 9,563,480. Washington, DC: U.S. Patent and Trademark Office.

Senyo, P. K., Addae, E., \& Boateng, R. (2018). Cloud computing research: A review of research themes, frameworks, methods, and future research directions. International Journal of Information Management, 38(1), 128-139. doi:10.1016/j.ijinfomgt.2017.07.007

Shaikh, R., \& Sasikumar, M. (2015). Data Classification for achieving Security in cloud computing. Procedia Computer Science, 45(C), 493-498. doi:10.1016/j.procs.2015.03.087

Shameem, P. M., \& Shaji, R. S. (2013). A methodological survey on load balancing techniques in cloud computing. IACSIT International Journal of Engineering and Technology, 4(5), 3801-3812.

Srivastava, P., \& Khan, R. (2018). A review paper on cloud computing. International Journal of Advanced Research in Computer Science and Software Engineering, 8(6), 17-20. doi:10.23956/ijarcsse.v8i6.711

Stergiou, C., Psannis, K. E., Kim, B. G., \& Gupta, B. (2018). Secure integration of IoT and cloud computing. Future Generation Computer Systems, 78, 964-975. doi:10.1016/j.future.2016.11.031 
Vivek Kumar Sehgal (SM'18) received the B.Tech. degree in Instrumentation Engineering from Sant Longowal Institute of Engineering and Technology (Deemed University), in 2000, M.Tech. degree in Process Control Engineering from Netaji Subhash Institute of Technology (Delhi University), in 2002, and the Ph.D. degree in Computer Science and Engineering from Uttrakhand Technical University, Dehradun, in 2007- 2010. He is currently an Associate Professor with the Department of Information Technology, Jaypee University of Information Technology, Solan, H.P-India. He is a Senior Member of IEEE, Senior Member of ACM, Senior member IEEE Computer Society, Member of IEEE Exe. Com. Delhi Section, member of SIAM, and a Fellow of Institution of Engineers. His current research interests include embedded processor architecture, hardware-software co-design, Networks-on-Chip, Smart Instrumentation, Cyber-Physical Systems, and Internet of Things. He is a member of the technical program committees for several technical conferences and editorial member of IEEE Access and other reputed journals. 